\title{
A bis-histidine-ligated unfolded cytochrome $c$ immobilized on anionic SAM shows pseudo-peroxidase activity
}

\author{
Antonio Ranieri a , Gianantonio Battistuzzi a , Marco Borsari a,*, Carlo Augusto Bortolotti a , Giulia Di Rocco a , \\ Stefano Monari a , Marco Sola ${ }^{\mathrm{a}, \mathrm{b}, *}$ \\ a Department of Chemistry, University of Modena and Reggio Emilia, via Campi 183, 41125 Modena, Italy \\ b CNR-INFM National Center nanoStructures and bioSystems at Surfaces, S3, Via Campi 213/A, I-41125 Modena, Italy
}

\section{A R T I C L E I N F O}

Article history:

Received 12 September 2011

Received in revised form 21 October 2011

Accepted 24 October 2011

Available online 29 October 2011

\section{Keywords:}

Cytochrome

Peroxidase activity

Self-assembled monolayer

Urea

Unfolding

\begin{abstract}
A B S T R A C T
Urea-unfolded yeast iso-1-cytochrome $c$ electrostatically adsorbed on a gold electrode coated with an anionic self-assembled monolayer yields a heme-mediated electrocatalytic reduction of $\mathrm{H}_{2} \mathrm{O}_{2}$ (pseudo-peroxidase activity). Under the same conditions, native cytochrome $c$ is inactive. In the unfolded protein, the Met80 heme iron ligand is replaced by a histidine residue yielding a bis-His-ligated form. $\mathrm{H}_{2} \mathrm{O}_{2}$ electrocatalysis occurs with an efficient mechanism likely involving direct $\mathrm{H}_{2} \mathrm{O}_{2}$ interaction with the iron(II) center and formation of a transient ferryl group. Comparison of the catalytic activity of a few urea-unfolded single and double Lys-to-Ala variants shows that the kinetic affinity of $\mathrm{H}_{2} \mathrm{O}_{2}$ for the heme iron and $\mathrm{k}_{\text {cat }}$ of the bis-His-ligated form are strongly affected by the geometry of protein adsorption, controlled by specific surface lysine residues.
\end{abstract}

(C) 2011 Elsevier B.V. All rights reserved.

\section{Introduction}

Mitochondrial cytochrome $c$ (cytc) is an important single-centered heme protein acting as a redox carrier in respiratory electrontransport chains [1-3]. Most of the protein is free or loosely bound to the inner mitochondrial membrane (IMM). However, approximately $15 \%$ of cytc is tightly bound to IMM and involved in events that initiate the apoptotic process [3-5]. In fact, cardiolipin (a phospholipid present in IMM) interacts with cytc inducing the release of the protein into the cytosol. Upon cardiolipin binding, cytc undergoes conformational changes which alter the environment of the heme pocket and induce a peroxidase activity [6,7]. Cytc can then oxidize cardiolipin which in turn triggers a cascade of events that bring about cell death. A nonnative cytc conformation imparted with peroxidase activity has been suggested to be also involved in several degenerative diseases and represents an additional source of oxidative stress $[3,8]$. Although the role of the peroxidase activity of unfolded cytc is well established in several physiological and pathological contexts, the mechanistic details of the process and the structure of the unfolded species remain largely unknown. Previous studies showed the presence of two distinct states of surface-immobilized unfolded cytc differing mainly in the axial ligation of the heme center [9]. In particular, a native-like state is observed under mild unfolding conditions, in which the heme is still axially coordinated by Met80 and His18. At larger unfolding agent concentrations, a

\footnotetext{
* Corresponding authors. Fax: + 39059343543.

E-mail addresses: marco.borsari@unimore.it (M. Borsari), marco.sola@unimore.it (M. Sola).
}

second state prevails in which the Met80 ligand is replaced by a histidine residue. Here, we report on the catalytic properties of unfolded cytc electrostatically immobilized on a gold electrode coated with a negatively charged self-assembled monolayer (SAM). This work demonstrates that immobilized urea-unfolded cytc with a bis-His heme iron axial ligation is able to catalytically reduce $\mathrm{H}_{2} \mathrm{O}_{2}$, most probably through a peroxidase-like mechanism. While the peroxidase activity of non-native cytochrome $c$ towards cardiolipin in early apoptotic events is well known, the finding that an immobilized unfolded cytochrome $c$ in a bis-His ligated form is effective in the hydrogen peroxide reduction and, consequently, could play a role in cell oxidative stress, is unprecedented. Experiments on protein variants allow to stress the role of the lysine residue topography in the observed peroxidase properties.

\section{Experimental}

11-mercapto-1-undecanoic acid (MUA), 11-mercapto-1-undecanol (MU) and urea were from Sigma-Aldrich. Wild-type recombinant untrimethylated yeast iso- 1 cytochrome $c$ (ycc) and its variants K73A, K79A, K72AK73A, K72AK73AK79A were expressed and purified following the procedure described elsewhere [10]. A Potentiostat/Galvanostat PAR 273A was used to perform cyclic voltammetry (CV) experiments. A polycrystalline gold wire was used as a working electrode, a Pt sheet and a saturated calomel electrode as counter and reference electrode, respectively. The working gold electrode was functionalized as reported elsewhere [9]. CV experiments on the protein-coated electrodes were performed using a working solution containing $9 \mathrm{M}$ urea, $10 \mathrm{mM} \mathrm{NaClO}_{4}$ and $5 \mathrm{mM}$ phosphate buffer at 


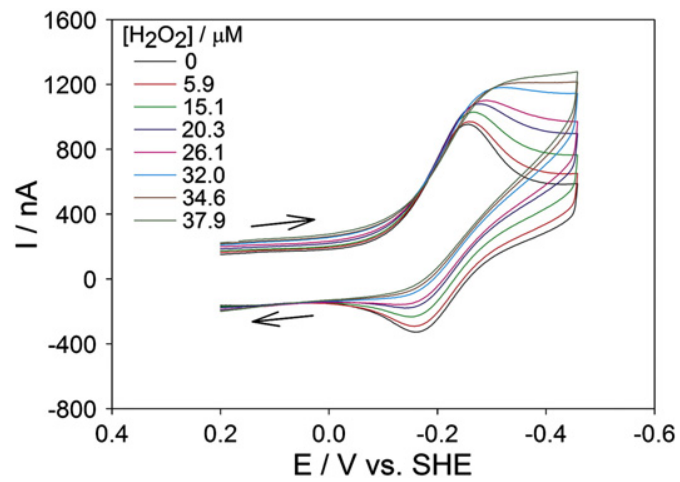

Fig. 1. Cyclic voltammograms for unfolded bis-His-ycc adsorbed on a gold electrode coated with $\mathrm{MUA} / \mathrm{MU}$ at increasing $\mathrm{H}_{2} \mathrm{O}_{2}$ concentration. Working solution: $10 \mathrm{mM}$ $\mathrm{NaClO}_{4}, 5 \mathrm{mM}$ phosphate buffer, and $9 \mathrm{M}$ urea. Sweep rate $0.05 \mathrm{~V} \mathrm{~s}^{-1}, \mathrm{pH} 7, \mathrm{~T}=293 \mathrm{~K}$.

pH 7 and $298 \mathrm{~K}$. The electrocatalytic reduction of $\mathrm{H}_{2} \mathrm{O}_{2}$ given by the urea-unfolded immobilized ycc and its variants was studied by gradually adding aliquots of a $\mathrm{H}_{2} \mathrm{O}_{2}$ solution at known concentration to the above solution under argon atmosphere. Surface coverages $\Gamma$ were found to be the same within the experimental error: $\Gamma=18.5 \pm$ $0.8 \mathrm{pmol} / \mathrm{cm}^{2}$.

\section{Results and discussion}

The cyclic voltammograms for bis-His-ligated ycc ( 9 M urea) electrostatically immobilized on MUA/MU recorded at increasing concentration of $\mathrm{H}_{2} \mathrm{O}_{2}$ at $\mathrm{pH} 7$ are shown in Fig. 1. The cathodic (anodic) currents progressively increase (decrease) with increasing $\mathrm{H}_{2} \mathrm{O}_{2}$ concentration, with a typical catalytic behavior, indicating that the adsorbed proteins catalyze $\mathrm{H}_{2} \mathrm{O}_{2}$ reduction (pseudo-peroxidase activity) [11]. The electrocatalytic effect is observed up to $\mathrm{H}_{2} \mathrm{O}_{2}$ concentration of about $40 \mu \mathrm{M}$. Below this concentration threshold, elimination of hydrogen peroxide by rinsing the electrode with buffer restored the CV signal of the protein. The catalytic currents could then be re-obtained upon $\mathrm{H}_{2} \mathrm{O}_{2}$ addition.

Therefore, under these conditions the protein layer is stable and re-usable. For substrate concentrations higher than $40 \mu \mathrm{M}$, the cathodic currents irreversibly decrease and vanish for $\mathrm{H}_{2} \mathrm{O}_{2}$ concentration above $60 \mu \mathrm{M}$. The variants showed the same behavior. In all cases, as above, the CV signal of the protein is observed again after peroxide removal. Therefore the current decrease cannot be ascribed to protein denaturation or desorption from the electrode, but at these

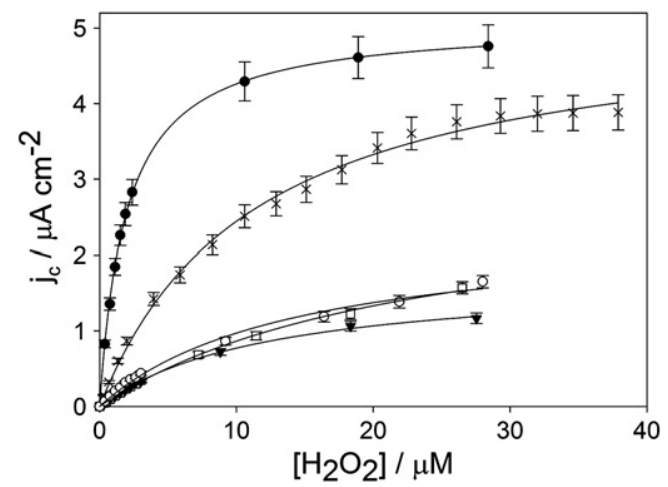

Fig. 2. Michaelis-Menten plot made with the electrocatalytic current densities $\left(\mathrm{j}_{\mathrm{c}}\right)$ yielded by unfolded bis-His-ycc and its variants adsorbed on a gold electrode coated with MUA/MU at increasing $\mathrm{H}_{2} \mathrm{O}_{2}$ concentration: ycc $(\times)$; K73A ( $\left.\square\right)$; $\mathrm{K} 79 \mathrm{~A}(\bullet)$; K72AK73A ( $\mathbf{\nabla})$; K72AK73AK79A (O). Working solution: $10 \mathrm{mM} \mathrm{NaClO}_{4}, 5 \mathrm{mM}$ phosphate buffer, and $9 \mathrm{M}$ urea. Sweep rate $0.05 \mathrm{~V} \mathrm{~s}^{-1}, \mathrm{pH} 7, \mathrm{~T}=293 \mathrm{~K}$. peroxide concentrations the reduction reaction most likely involves different intermediates.

All the immobilized proteins in their native His-Met-ligated state are unable to carry out the reductive electrocatalysis of $\mathrm{H}_{2} \mathrm{O}_{2}$. Therefore, it is the bis-His ligated ycc conformer, which prevails at large unfolding agent concentrations [9], that is responsible for the observed pseudo-peroxidase activity. Moreover, the CV measurements show that the cathodic peak potential does not change appreciably with increasing $\mathrm{H}_{2} \mathrm{O}_{2}$ concentration (Fig. 1). This indicates that the catalytically active ycc state, namely that which interacts with the $\mathrm{H}_{2} \mathrm{O}_{2}$ substrate, is the reduced state. Although a six-coordinated heme iron center lacks the interaction sites for the $\mathrm{H}_{2} \mathrm{O}_{2}$ ligand, the reduced form of the bis-His-ligated species is known to be unstable and to lose one of the histidine ligands from the iron(II) center [9]. In this way, an axial site of the metal center becomes available for the interaction with $\mathrm{H}_{2} \mathrm{O}_{2}$. Moreover, recent literature shows that in several reactions iron (II) is oxidized to ferryl group by $\mathrm{H}_{2} \mathrm{O}_{2}$ [12]. It follows that a mechanism involving the reduced heme of the bisHis-ligated ycc conformer can be proposed which accounts for the observed electrocatalytic activity:

$$
\begin{aligned}
& \text { cytFe }(\mathrm{III})-\mathrm{His}+\mathrm{e} \rightarrow \text { cytFe }(\mathrm{II})---\mathrm{His} \\
& \text { cytFe }(\mathrm{II})---\mathrm{His}+\mathrm{H}_{2} \mathrm{O}_{2} \rightarrow \text { cytFe }(\mathrm{IV})=\mathrm{O}(\mathrm{His})_{\text {uncoord }}+\mathrm{H}_{2} \mathrm{O} \\
& \text { cytFe }(\mathrm{IV})=\mathrm{O}(\mathrm{His})_{\text {uncoord }}+\mathrm{e}+2 \mathrm{H} \rightarrow \text { cytFe}(\mathrm{III})-\mathrm{His}+\mathrm{H}_{2} \mathrm{O} .
\end{aligned}
$$

Since the heme ferryl group has a remarkably more positive $\mathrm{E}^{\circ}$ value than the ferric heme, under the conditions of the catalytic event, it is immediately reduced at the potential of the electrode, i.e. along with the reduction of the cytFe(III)-His species.

The catalytic activity of electrode-immobilized ycc and its Lys-to-Ala variants in $9 \mathrm{M}$ urea can be successfully estimated from the MichaelisMenten equation. In fact, although the step involving the formation of $\mathrm{Fe}(\mathrm{IV})$ species is irreversible, it is well established that this step is preceded by the formation of one or more reversible enzyme-substrate intermediates (a hydroperoxo complex) [13-15]. Therefore, in this respect, the overall mechanism follows the Michaelis-Menten model, but the corresponding analysis of the catalytic activity provides information on a composite dominant process involved in the catalytic kinetics. As a consequence, the catalytic constants $\mathrm{K}_{\mathrm{M}}$ and $\mathrm{V}_{\max }$ are composite quantities and acquire the status of "apparent".

The Michaelis-Menten equation can be expressed in terms of current density $[11,16-18]$ :

$j_{c}=\frac{j_{\max }\left[\mathrm{H}_{2} \mathrm{O}_{2}\right]}{\mathrm{K}_{\mathrm{M}}+\left[\mathrm{H}_{2} \mathrm{O}_{2}\right]}$

where $j_{\mathrm{c}}$ is the electrocatalytic current density (taken as the ratio of the difference between the cathodic peak current in the presence and in the absence of a given concentration of hydrogen peroxide and the electrochemically determined area of the electrode), $j_{\max }$ is the maximum current density at substrate saturation. The Michaelis

Table 1

Kinetic parameters for electrocatalytic $\mathrm{H}_{2} \mathrm{O}_{2}$ reduction carried out by the urea-unfolded bis-His-ligated form of ycc and its variants adsorbed on gold electrode coated with MUA/MU.

\begin{tabular}{lcl}
\hline protein & $\mathrm{K}_{\mathrm{M}} / \mu \mathrm{M}$ & $\mathrm{J}_{\max } / \mu \mathrm{Acm}^{-2}$ \\
\hline ycc & 9.3 & 5.18 \\
K79A & 2.0 & 5.50 \\
K73A & 18.8 & 2.68 \\
K72AK73A & 18.3 & 5.53 \\
K72AK73AK79A & 11.5 & 2.34
\end{tabular}

a Working solution: $10 \mathrm{mM} \mathrm{NaClO}_{4}, 5 \mathrm{mM}$ phosphate buffer and $9 \mathrm{M}$ urea. $\mathrm{pH} 7$, $\mathrm{T}=293 \mathrm{~K}$. The upper values for the errors (standard deviation) on $j_{\max }$ and $\mathrm{K}_{\mathrm{M}}$ are $\pm 0.22 \mu \mathrm{A} \mathrm{cm}^{-2}$ and $\pm 0.9 \mu \mathrm{M}$, respectively. 
constant $K_{M}$ is an indicator of the kinetic affinity between the protein and substrate: a smaller $K_{M}$ value indicates higher protein affinity for $\mathrm{H}_{2} \mathrm{O}_{2}$. The plots obtained at pH 7 (Fig. 2) yield the $j_{\text {max }}$ and $\mathrm{K}_{\mathrm{M}}$ values listed in Table 1.

These data are indicative of a large catalytic efficiency for all the immobilized protein in the presence of high concentration of unfolding agent. It is worthy of note that the $\mathrm{K}_{\mathrm{M}}$ values of the investigated proteins are in the micromolar range (Table 1) as that for M80A variant of ycc, in which the axial ligand Met80 is substituted by an $\mathrm{OH}^{-}$ ion $[11,17]$. Therefore, the kinetic affinity of $\mathrm{H}_{2} \mathrm{O}_{2}$ for the heme iron in these systems is from one to three orders of magnitude larger than those for peroxidases [19,20], hemoglobin [21-23], microperoxidase 11 [24-26] and wt cytochrome $c[18,27]$ immobilized on modified electrodes. We also observe that $\mathrm{K}_{\mathrm{M}}$ follows the order: $\mathrm{K} 73 \mathrm{~A} \approx \mathrm{K} 72 \mathrm{~A}-$ K73A $>$ K72AK73AK79A $>$ ycc $>$ K79A. Replacement of Lys79 with an Ala residue in the bis-His-ligated species remarkably increases the affinity of $\mathrm{H}_{2} \mathrm{O}_{2}$ towards the heme center, while replacement of Lys73 yields the opposite effect.

The structure of unfolded bis-His-ligated ycc is not available presently, but it is well known that these lysine residues play a relevant role in the electrostatic immobilization of the protein $[28,29]$. We can therefore assume that these lysine residues are also involved in the interaction of bis-His-ligated ycc with the SAM surface. As a consequence, the opposite change in $\mathrm{K}_{\mathrm{M}}$ observed for $\mathrm{K} 73 \mathrm{~A}$ and $\mathrm{K} 79 \mathrm{~A}$ point mutants compared to wt ycc could be due to a different accessibility of the heme center to substrate due to a different adsorption geometry controlled by surface lysines.

In Eq. (4) $j_{\max }$ is the electrochemical equivalent of $\mathrm{v}_{\max }$ in the conventional Michaelis-Menten model. As such, it depends on $\mathrm{k}_{\text {cat }}$ and it is proportional to the surface concentration of the catalytically active protein on the electrode. We observe that the $j_{\max }$ value for ycc and K79A is larger than that for K73A, K72AK73A, K72AK73AK79A. As the protein coverage of the SAM is conserved for all the investigated proteins, this difference in $j_{\max }$ must reflect differences in $\mathrm{k}_{\text {cat }}$.

\section{Conclusions}

In this work, we have shown that the bis-His-ligated conformer of urea-denatured ycc immobilized electrostatically on a SAM-coated gold electrode is able to carry out efficiently the electrocatalytic reductive turnover of $\mathrm{H}_{2} \mathrm{O}_{2}$. This study adds new elements for understanding the redox chemistry of unfolded cytochrome $c$ which, once released in the cellular cytosol in the early stages of the apoptotic process, exerts a peroxidase activity that contributes to the oxidative stress $[3,4,8]$. The present data suggest that in the presence of an electron donor, unfolded cytc may also possess a detoxifying role carrying out the two-electron reduction of $\mathrm{H}_{2} \mathrm{O}_{2}$ to water. In addition, this work could open the way to the development of electrocatalytic hybrid unfolded cytc-modified electrode surfaces for $\mathrm{H}_{2} \mathrm{O}_{2}$ biosensing in the clinical and pharmaceutical areas.

\section{Acknowledgments}

This work was supported by a grant from the MIUR of Italy (PRIN 2009 Prot. 20098Z4M5E_002) and by the Fondazione Cassa di Risparmio di Modena (progetto di Ricerca Internazionale Prot. 1297.08.8C).

\section{References}

[1] R.A. Scott, G.A. Mauk, Cytochrome c: A Multidisciplinary Approach, University Science Books, Sausalito CA, 1996.

[2] G.R. Moore, G.W. Pettigrew, Cytochromes c: Evolutionary, Structural, and Physicochemical Aspects, Springer-Verlag, Berlin, 1990.

[3] R. Santucci, F. Sinibaldi, A. Patriarca, D. Santucci, L. Fiorucci, Expert Review of Proteomics 7 (2010) 507

[4] Y.-L. Ow, D.R. Green, Z. Hao, T.W. Mak, Nature Molecular Cell Biology 9 (2008) 523.

[5] H. Bayir, B. Fadeel, M.J. Palladino, E. Witasp, I.V. Kurnikov, Y.Y. Tyurina, V.A. Tyurin, A.A. Amoscato, J. Jiang, P.M. Kochanek, S.T. DeKosky, J.S. Greenberger, A.A. Shvedova, V.E. Kagan, Biochimica et Biophysica Acta, Bioenergetics 1757 (2006) 648.

[6] F. Sinibaldi, L. Fiorucci, A. Patriarca, R. Lauceri, T. Ferri, M. Coletta, R. Santucci, Biochemistry 47 (2008) 6928.

[7] P. Ascenzi, R. Santucci, M. Coletta, F. Polticelli, Biophysical Chemistry 152 (2010) 21.

[8] H. Bayir, A.A. Kapralov, J. Jiang, Z.T. Huang, Y.Y. Tyurina, V.A. Tyurin, Q. Zhao, N.A. Belikova, I.I. Vlasova, A. Maeda, J.H. Zhu, H.M. Na, P.G. Mastroberardino, L.J. Sparvero, A.A. Amoscato, C.T. Chu, J.T. Greenamyre, V.E. Kagan, Journal of Biological Chemistry 284 (2009) 15951.

[9] S. Monari, D. Millo, A. Ranieri, G. Di Rocco, G. van der Zwan, C. Gooijer, S. Peressini, C. Tavagnacco, P. Hildebrandt, M. Borsari, Journal of Biological Inorganic Chemistry 15 (2010) 1233.

[10] G. Battistuzzi, M. Borsari, F. De Rienzo, G. Di Rocco, A. Ranieri, M. Sola, Biochemistry 46 (2007) 1694

[11] S. Casalini, G. Battistuzzi, M. Borsari, C.A. Bortolotti, G. Di Rocco, A. Ranieri, M. Sola, The Journal of Physical Chemistry. B 114 (2010) 1698.

[12] H.B. Dunford, Coordination Chemistry Reviews 233-234 (2002) 311.

[13] T.L. Poulos, J. Kraut, Journal of Biological Chemistry 255 (1980) 8199

[14] P. Jones, B.H. Dunford, Journal of Inorganic Biochemistry 99 (2005) 2292.

[15] B.H. Dunford, Peroxidases and Catalases: Biochemistry, Biophysics, Biotechnology and Physiology, Wiley, Hoboken NJ, 2010.

[16] R.A. Kamin, G.S. Wilson, Analytical Chemistry 52 (1980) 1198.

[17] S. Casalini, G. Battistuzzi, M. Borsari, C.A. Bortolotti, A. Ranieri, M. Sola, The Journal of Physical Chemistry. B 112 (2008) 1555.

[18] H. Ju, S. Liu, B. Ge, F. Lisdat, F.W. Scheller, Electroanalysis 14 (2002) 141.

[19] D.J. Li, S.N. Tian, H. Ge, Analytica Chimica Acta 335 (1996) 137.

[20] Y. Xiao, H.X. Ju, H.Y. Chen, Analytical Biochemistry 278 (2000) 22.

[21] Y.-D. Zhao, Y.-H. Bi, W.-D. Zhang, Q.-M. Luo, Talanta 65 (2005) 489.

[22] C. Fan, H. Wang, S. Sun, D. Zhu, G. Wagner, G. Li, Analytical Chemistry 73 (2001) 2850.

[23] Z. Tong, R. Yuan, Y. Chai, S. Chen, Y. Xie, Thin Solid Films 515 (2007) 8054

[24] T. Lötzbeyer, W. Schuhmann, H.-L. Schmidt, Journal of Electroanalytical Chemistry 395 (1995) 339.

[25] T. Lötzbeyer, W. Schuhmann, H.-L. Schmidt, Sensors and Actuators B: Chemical 33 (1996) 50.

[26] A.N.J. Moore, E. Katz, I.J. Willner, Electroanalytical Chemistry 417 (1996) 189

[27] L. Wang, D.H. Waldeck, Journal of Physical Chemistry C 112 (2008) 1351.

[28] J. Zhou, J. Zheng, S.J. Jiang, Physical Chemistry B 108 (2004) 17418.

[29] J. Xu, E.F. Bowden, Journal of the American Chemical Society 128 (2006) 6813. 